\title{
Cuestionario de Clima Motivacional de la Clase: Validez Intercultural, Intergénero, Evolutiva y Predictiva
}

\author{
Classroom Motivational Climate Questionnaire: Intercultural, Inter-Gender, \\ Developmental and Predictive Validity
}

\author{
Francisco Leal-Soto ${ }^{1}$ y Jesús Alonso-Tapia ${ }^{2}$
}

\begin{abstract}
Resumen
Parte del trabajo docente consiste en crear un clima motivacional propicio para el aprendizaje. El Cuestionario de Clima Motivacional de la Clase (CMCQ) fue desarrollado para su evaluación. Se presentan dos estudios de validación del CMCQ. El primero sometió a prueba la invarianza factorial del instrumento entre estudiantes de educación secundaria españoles y chilenos $(n=793)$, y por sexo en estos últimos; el segundo sometió a prueba la invarianza factorial entre niveles educativos y la validez predictiva del CMCQ sobre cambio en variables motivacionales y rendimiento en estudiantes chilenos $(n=2701)$, a través de modelamiento con ecuaciones estructurales. Los resultados confirman la estructura unifactorial del instrumento y su invarianza por género y nivel educativo, pero muestran diferencias entre culturas; y confirman la validez predictiva del mismo respecto de cambio en variables motivacionales y, a través de éstas, sobre el rendimiento, aunque con variaciones entre culturas y niveles educativos.
\end{abstract}

Palabras clave: clima motivacional de clase, motivación, educación secundaria, rendimiento

\begin{abstract}
Part of teaching is to create a learning motivational climate. Classroom Motivational Climate Questionnaire (CMCQ) was developed for assess the motivational climate in the classroom. Two validation studies of CMCQ are presented. The first tested the factorial invariance of the instrument among Spanish and Chilean secondary school students $(n=793)$, and sex in the latter; the second tested the factorial invariance between educational levels and the predictive validity of CMCQ on change in motivational variables and achievement in Chilean students $(n=2701)$, through structural equation modeling. The results confirm the factor structure of the instrument and its invariance by gender and educational level, but show differences between cultures; and confirm the predictive validity of CMCQ for change in motivational variables and, through them, on performance, although with variations between cultures and educational levels.
\end{abstract}

Keywords: classroom motivational climate, motivation, highschool education, achievement

La realización de ambos estudios contó con apoyo financiero a través de GRANT 1110722, otorgado por FONDECYT al primer autor. Los autores agradecen el apoyo del Dr. Rodrigo Ferrer-Urbina en la revisión de los aspectos técnicos del reporte de los resultados en el manuscrito, y de la Srta. Daniela Ramírez en la revisión formal de las referencias.

${ }^{1}$ Dr. (c) en Psicología, académico del Departamento de Ciencias Sociales sede Esmeralda, Iquique, Universidad de Tarapacá, Chile, con domicilio en Covadonga 894, 1100723, Iquique, Chile. investigador principal del Centro de Investigación para la Educación Inclusiva, PIE160009. Tel.: +56572727250. Correo: fleal@uta.cl

${ }^{2}$ Dr. en Psicología, catedrático del Departamento de Psicología Biológica y de la Salud de la Facultad de Psicología de la Universidad Autónoma de Madrid, con domicilio en Campus Cantoblanco, C/Iván Pavlov, 6, 28049-Madrid, fono +34914974598, correo electrónico jesus.alonso@uam.es 


\section{Introducción}

Crear un ambiente que favorezca la disposición de los estudiantes hacia el aprendizaje y su compromiso con el mismo es una tarea esencial en el ambiente escolar (Patall, Vásquez, Steingut, Trimble, \& Pituch, 2016; Raghul \& Saradha, 2016). Aunque la motivación del estudiante por el aprendizaje también depende de factores personales y socioculturales, las características de la situación instruccional son relevantes, no sólo porque pueden moderar los efectos de los factores personales y socioculturales, sino porque la institución escolar, y particularmente los profesores, pueden actuar sobre ellas (Bucic \& Robinson, 2016; Høgheim \& Reber, 2016; Simón \& Alonso-Tapia, 2016; Villasana \& Alonso-Tapia, 2015) o establecer acciones específicas (García-Ripa, SánchezGarcía, \& Risquez, 2016). Meece, Anderman y Anderman (2006) reunieron abundante evidencia de que los ambientes escolares orientados hacia el aprendizaje, la comprensión y la adquisición de conocimientos y destrezas promueven patrones motivacionales y de aprendizaje positivos, mientras que ambientes escolares orientados a los resultados en sí mismos, como las notas o la exhibición de superioridad, si bien pueden estimular el rendimiento de algunos estudiantes, producen una disminución de la motivación en muchos otros. Ames (1992) acuñó el concepto clima motivacional de clase (CMC) para referirse al ambiente motivacional que propician las acciones de los profesores, distinguiendo entre climas orientados al aprendizaje (adquisición y dominio de conocimientos y competencias) y al rendimiento (calificaciones, resultados), promoviendo en los estudiantes metas que se asocian a diferentes resultados en motivación y rendimiento (Schwinger, Steinmayr, \& Spinath, 2016). Además, propuso el modelo TARGET, que incluye cinco dimensiones de las actividades en los que los profesores pueden actuar para mejorar la motivación de los estudiantes: tipo de tarea, formas de ejercer la autoridad, administración de los reconocimientos, formas de agrupar para el trabajo, tipo de evaluación y administración del tiempo. Una conocida revisión de Urdan y Turner (2005) recogió un conjunto de evidencia que respalda la importancia de los factores mencionados para la motivación de los estudiantes hacia el aprendizaje. No obstante, también hay evidencia de que los mismos no impactan de igual manera a todos los estudiantes o grupos de estudiantes (Alonso-Tapia, Huertas, \& Ruiz, 2010; Alonso-Tapia, Nieto, \& Ruiz, 2013; Alonso-Tapia, Panadero, \& Ruiz, 2014; AlonsoTapia, \& Simón, 2012; Núñez, Vallejo, Rosario, Tuero, \& Valle, 2014; Salili \& Hoosain, 2007). Por esta razón, el conocimiento que pueda obtener el profesor sobre los factores que más impactan a sus propios estudiantes puede hacer gran diferencia en lo que logre con ellos, pues le permite concentrar sus esfuerzos de motivación en los más relevantes. Alonso-Tapia y sus colaboradores han identificado un conjunto de 16 pautas de actividad docente coherentes con el modelo TARGET y desarrollaron el Cuestionario de Clima Motivacional de la Clase (CMCQ) para evaluar su influencia en el CMC percibido por los estudiantes (Alonso-Tapia \& Fernández, 2008). El CMCQ ha mostrado muy buenas características psicométricas para identificar los diferentes patrones que contribuyen a configurar un CMC orientado al aprendizaje y predecir su efecto en el cambio en variables motivacionales de los estudiantes, como interés, capacidad percibida, esfuerzo, expectativas de éxito y satisfacción con el profesor (Alonso-Tapia \& Fernández, 2008, 2009; Alonso-Tapia \& Moral, 2010; Alonso-Tapia \& Simón, 2012; Villasana \& Alonso-Tapia, 2015), así como su relación con otras variables relevantes en el desempeño estudiantil, como resiliencia y autorregulación (Alonso-Tapia et al., 2014; Alonso-Tapia, Nieto, \& Ruiz, 2013). Asimismo, posee evidencia de validez transcultural, manteniéndose la estructura unifactorial entre estudiantes españoles, mexicanos y franceses, así como en inmigrantes residentes en España (Alonso-Tapia \& Fernández, 2009; Alonso-Tapia \& Moral, 2010; Alonso-Tapia \& Simón, 2012; Villasana \& Alonso-Tapia, 2015). No obstante, se observan algunas diferencias poblacionales leves en los pesos de medida al configurar el CMC. Por ejemplo, el estudio de Alonso-Tapia y Simón (2012) muestra que en poblaciones inmigrantes el clima se refleja menos en dimensiones directamente relacionadas con las tareas instruccionales (como usar la novedad o dar instrucciones paso a paso) que en 
los españoles, en quienes se refleja más en la relación entre el profesor y el estudiante (como estimular la participación y preocuparse por cada alumno), lo que da cuenta de la diversa importancia que estudiantes de distintas culturas le asignan a cada uno de los patrones de actuación docente incluidos en el CMCQ como moduladores motivacionales.

Del mismo modo, los estudios iniciales mostraron que la invarianza factorial de los pesos de medida se mantenía entre estudiantes de secundaria y bachillerato $\left(7^{\circ}-10^{\circ}\right.$ y $11^{\circ}-12^{\circ}$ grados) de una misma cultura (Alonso-Tapia \& Fernández, 2008, 2009). No obstante, en muchos países, incluidos España y Chile, la enseñanza secundaria tiene ciclos o subdivisiones que responden a formas de ordenamiento curricular diversas e involucran ciclos vitales en que hay mucha variabilidad, lo que podría marcar diferencias en la forma en que los estudiantes perciben su entorno escolar. Por esta razón, los hallazgos en un sistema podrían no replicarse en otro con subdivisiones diferentes. Es el caso entre Chile y España. En España, la Enseñanza Secundaria Obligatoria cubre los grados $7^{\circ}$ a $10^{\circ}$, siendo el último un curso propedéutico que da paso a los dos grados siguientes, no obligatorios, que constituyen el bachillerato (Ministerio de Educación, Cultura y Deporte, 2004, s.f.). En Chile, la Ley General de Educación establece la enseñanza obligatoria hasta el $12^{\circ}$ grado, situando la secundaria entre los grados $7^{\circ}$ y $12^{\circ}$ (Ministerio de Educación, 2009); no obstante, hasta el presente los primeros dos niveles (grados $7^{\circ}$ y $8^{\circ}$ ) siguen situados en la Enseñanza General Básica, según establece la antigua Ley Orgánica Constitucional de Enseñanza (Ministerio de Educación, 1990), y, por tanto, son impartidos tanto en escuelas que ofrecen sólo nivel primario como en aquellas que ofrecen también la educación secundaria.

En relación al género, los resultados han sido menos concluyentes. Alonso-Tapia y Fernández (2008), Fernández (2004) y Villasana y AlonsoTapia (2015) no reportan diferencias por género, pero Alonso-Tapia y Fernández (2009), aunque replican la estructura unifactorial en ambos sexos, encontraron diferencias en la importancia relativa de cada patrón de conducta docente en la configuración del CMC. Habida cuenta de la variabilidad en el peso que diferentes grupos culturales y estudiantes de diferentes niveles educativos les asignan a los indicadores, y los resultados no concluyentes sobre la influencia del sexo, parece recomendable determinar estas variaciones antes de utilizar el instrumento en un contexto diferente. Por otra parte, aunque el CMC ha mostrado ser un buen predictor de cambio en variables motivacionales, su relación con el rendimiento escolar ha sido poco explorada. Alonso-Tapia y Moral (2010) encontraron una correlación estadísticamente significativa pero de baja magnitud ( $r=.272, p<.01)$ entre CMCQ y rendimiento en una pequeña muestra de estudiantes adultos no universitarios, pero esta relación no ha sido explorada en estudiantes regulares del ciclo secundario o de bachillerato.

Con el interés de disponer del CMCQ para trabajar en el contexto educativo chileno, se presentan aquí dos trabajos en los cuales se aborda la validez intercultural del CMCQ comparando resultados en estudiantes de bachillerato españoles y chilenos, su invarianza factorial entre niveles educativos y por género en el contexto chileno, y la relación entre el CMCQ y el rendimiento en estudiantes de educación secundaria. De acuerdo a los antecedentes previos, se hipotetizó que la estructura unifactorial sería confirmada tanto entre culturas como entre niveles educativos y sexo; que se encontraría algunas diferencias en la forma en que se configura el CMC entre los diferentes grupos; que el CMCQ tendría validez predictiva respecto al cambio en variables motivacionales; y que el CMCQ tendría una relación significativa pero baja con el rendimiento.

\section{Estudio 1: Validación transcultural del CMCQ en estudiantes españoles y chilenos}

Aunque estudios previos mostraron la validez del CMCQ en estudiantes españoles (AlonsoTapia y Fernández, 2008), mexicanos (AlonsoTapia y Fernández, 2009) y franceses (Villasana y Alonso-Tapia, 2015), las diferencias encontradas en los pesos de medida que los distintos grupos confieren a las dimensiones del CMC reflejan diferentes énfasis en la configuración del mismo. Este hecho aconseja determinar esta estructura antes de trabajar con el instrumento de manera aplicada en otros países -por ejemplo, para determinar las características motivacionales 
atribuidas por los estudiantes a un profesor- pues éstas tendrán diferente significado dependiendo de la importancia relativa que los patrones docentes que constituyen el CMC evaluado por el CMCQ tengan en cada contexto cultural. En este estudio, se sometió a prueba la validez transcultural del CMCQ en el contexto de educación secundaria chilena, comparándolo con la muestra original española equivalente, correspondiente al ciclo de bachillerato; y se exploró la invarianza entre géneros en la muestra chilena.

\section{Método}

\section{Participantes}

Los participantes fueron 793 estudiantes chilenos de secundaria $\left(11^{\circ}\right.$ y $12^{\circ}$ grados $), 46.9 \%$ mujeres y con una media de edad de 16.75 años ( $D T=.777) ; \quad$ y 711 estudiantes españoles de bachillerato, 67.9\% mujeres, con 16.95 años de edad media $(D T=1.398)$. Fueron seleccionados por conveniencia en escuelas que tuvieron disposición a colaborar. La muestra chilena fue dividida aleatoriamente en dos grupos $\left(n_{1}=424\right.$, $\mathrm{n}_{2}=369$ ), el primero para validar inicialmente el modelo y el segundo para su validación cruzada.

\section{Instrumentos}

El Cuestionario de Clima Motivacional de la Clase CMCQ de Alonso-Tapia y Fernández (2008) consta de 32 ítems que miden la apreciación de los estudiantes respecto del uso de 16 pautas docentes con efectos motivacionales. Cada pauta es evaluada por dos ítems, uno redactado en sentido positivo y uno redactado en sentido negativo, para evitar el efecto de aquiescencia al responder ("A menudo, este profesor nos presenta información nueva $\mathrm{O}$ sorprendente que despierta nuestro interés"; "El profesor de esta asignatura no detiene su explicación para ayudar a los alumnos que no le entienden" [-]). El formato de respuesta es escala Likert de cinco puntos, desde completo desacuerdo hasta completo acuerdo. Los índices de consistencia interna de la escala completa han sido sobre .90 ( $\alpha$ de Cronbach) (Alonso-Tapia \& Fernández, 2008, 2009; Villasana \& AlonsoTapia, 2015) y hasta .98 ( $\omega$ de McDonald, 1999; Alonso-Tapia, Ruiz, \& Huertas, 2016). Siguiendo el modelo de validación de los estudios previos, se utilizaron cinco escalas desarrolladas por AlonsoTapia y Fernández (2008) para medir: a) el grado en que el estudiante atribuye al profesor cambios en diferentes aspectos de su propia motivación: en las expectativas de resultado ("Debido a la forma en que enseña este profesor, veo difícil obtener la nota que deseo" [-]); en el interés por la materia ("Lo bueno de este profesor es que hace que me interese por lo que enseña"); en la habilidad percibida ("Este profesor hace que mi capacidad para aprender esta materia sea cada vez mejor"); y en el esfuerzo dedicado ("Este profesor hace que se me quiten las ganas de esforzarme por aprender su asignatura" [-]; y b) su satisfacción con el propio profesor ("Si se pudiera elegir profesor, aconsejaría a mis compañeros que eligieran a éste, sin dudarlo"). Las cuatro escalas de percepción de cambio motivacional incluyen tres ítems y la de satisfacción cuatro, todas en formato tipo Likert de cinco puntos. La consistencia interna de estas escalas es aceptable, con coeficientes $\alpha$ de Cronbach entre .66 y .80 (Villasana \& AlonsoTapia, 2015), y $\omega$ entre .91 y .94 (Alonso-Tapia, Ruiz, \& Huertas, 2016).

\section{Procedimiento}

El estudio se enmarca en un programa de investigación que ha sido aprobado por el comité de ética de investigación institucional. Además, se obtuvo autorización de las escuelas y se solicitó autorización por escrito a los padres de los estudiantes, quienes consintieron en participar voluntariamente. Los datos fueron recogidos por ayudantes de investigación en el transcurso de los años lectivos 2012-2013, en sesiones que duraron entre 45 y 90 minutos, dentro del horario regular de clases de los estudiantes. Se instruyó a los estudiantes que respondieran respecto de una asignatura y profesor en particular.

\section{Análisis de datos}

Se realizó un análisis factorial confirmatorio (AFC) usando estimación de máxima verosimilitud con la primera submuestra chilena (AFC-1), y luego una serie de AFC multigrupo considerando: validación cruzada entre los dos subgrupos aleatorios de estudiantes chilenos (AFC-2); género en la muestra chilena completa (AFC-3); y entre la muestra chilena y española 
(AFC-4). Para juzgar el ajuste de los modelos, se utilizaron los indicadores $\chi^{2}, \chi^{2} / \mathrm{gl}$, GFI, IFI, CFI y RMSEA, con los criterios de ajuste sugeridos por Hair, Black, Babin y Anderson (2010). Para analizar diferencias entre saturaciones factoriales en los AFC en los que resultó pertinente, se utilizó el estadístico Z de Clogg, Petkova y Haritou (1995). Para obtener confiabilidad de las escalas se utilizaron los coeficientes Alpha de Cronbach y Omega de McDonald (1999). Finalmente, se obtuvo la correlación bivariada entre las variables en la muestra chilena completa.

\section{Resultados}

Análisis factorial confirmatorio, muestra chilena, grupo 1 (AFC-1). La Figura 1 muestra los correspondientes valores estimados estandarizados del modelo propuesto, y la Tabla 1 muestra los indicadores de ajuste del mismo (AFC-1). Todos los pesos estimados fueron significativos $(p<.001)$. El estadístico Ji-cuadrado es significativo, probablemente debido al tamaño de la muestra, pero los restantes índices de ajuste se encuentran dentro de los límites normalmente utilizados como criterio de aceptación de un modelo.

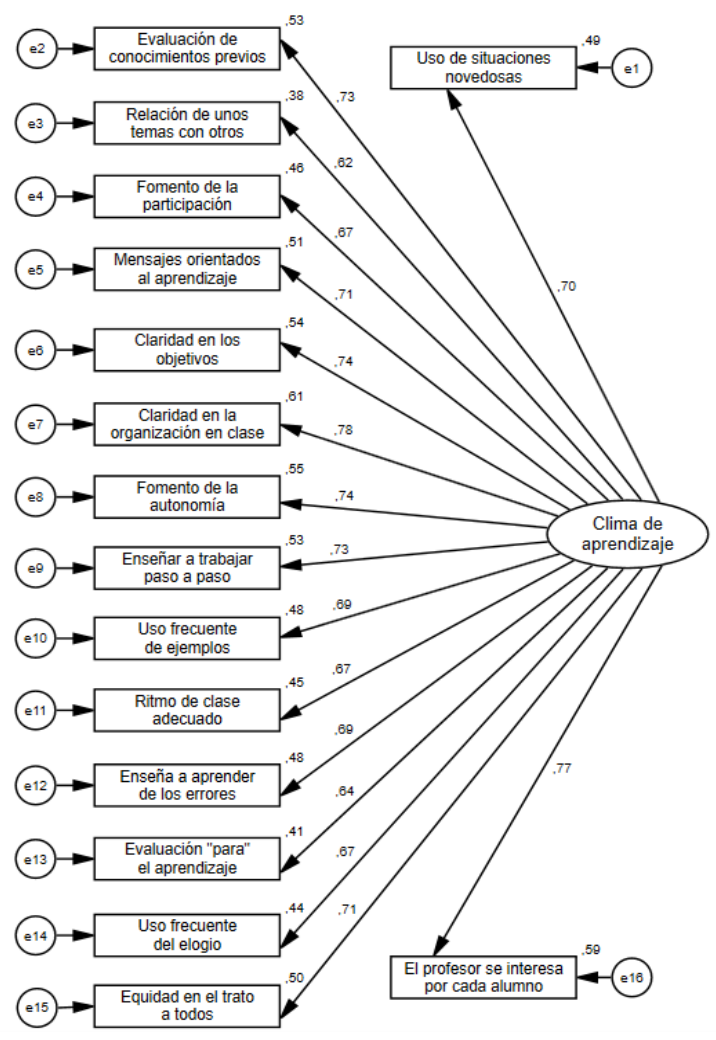

Figura 1. CMCQ. Solución confirmatoria estandarizada en la muestra chilena, grupo 1.
Análisis factorial confirmatorio multigrupo, validación cruzada entre subgrupos de la muestra chilena (AFC-2). Para comprobar la validez del modelo se realizó un análisis multigrupo. En este análisis (AFC-2, Tabla 1), los índices de ajuste se encuentran también dentro de límites aceptables. Además, el ajuste no se reduce de modo significativo, aunque se impongan restricciones de igualdad entre los diferentes parámetros.

Análisis factorial confirmatorio multigrupo por género, Chile (AFC-3). Para comprobar si el ajuste del modelo variaba en función del género se realizó un análisis multigrupo por géneros. En este análisis (AFC-3, Tabla 1), los índices de ajuste se encuentran también dentro de límites aceptables. Además, el ajuste no se reduce de modo significativo, aunque se impongan restricciones de igualdad entre los diferentes parámetros.

\section{Análisis factorial confirmatorio multigrupo por} país, Chile-España (AFC-4). Para comprobar si el ajuste del modelo variaba en función del país, se realizó un análisis multigrupo por países. En este análisis (AFC-4, Tabla 1), los índices de ajuste se encuentran también dentro de límites aceptables. Sin embargo, en el AFC-4, aunque en ambos países los datos se ajustan al modelo teórico, hay diferencias significativas entre las pendientes si se ponen restricciones sobre la igualdad de los pesos de medida $\left(\chi^{2}\right.$ chile-esp $=89.88$, $g l=15, p>.001)$, diferencia que significa que el grado de saturación de cada variable en la escala es diferente. Por este motivo, es necesario preguntarse en qué variables se da la diferencia, para lo que se utilizó el estadístico Z de Clogg, Petkova y Haritou (1995). Estas comparaciones mostraron que los estudiantes chilenos otorgan mayor peso al uso de situaciones novedosas, la evaluación del conocimiento previo, relacionar los temas, entregar mensajes orientados al aprendizaje, la claridad de objetivos y de la organización de las actividades, el trabajo por pasos, el uso de ejemplos, el uso de la evaluación como instrumento de aprendizaje y la equidad.

\section{Consistencia interna y correlaciones}

Los índices de consistencia interna $\alpha$ de Cronbach y $\omega$ de McDonald de las escalas para la 
Tabla 1. Estadísticos de bondad de ajuste para el modelo base del CMC, para el análisis de validación cruzada, y para los análisis multigrupo por género (Chile) y por país (Chile-España)

\begin{tabular}{ccccccccc}
\hline Modelo & $\chi^{2}$ & $g l$ & $p$ & $\chi^{2} / g l$ & GFI & IFI & TLI & RMSEA \\
\hline AFC-1 & 246.77 & 104 & .000 & 2.37 & .93 & .96 & .96 & .057 \\
AFC-2 & 496.07 & 240 & .000 & 2.06 & .93 & .96 & .96 & .039 \\
AFC-3 & 482.92 & 240 & .000 & 2.01 & .93 & .94 & .94 & .038 \\
AFC-4 & 698.86 & 210 & .000 & 3.32 & .94 & .96 & .96 & .039 \\
\hline \multicolumn{7}{l}{ Nota. AFC-1: Modelo base $(n=424) ;$ AFC-2: Cross Validación $(n=424,369) ;$ AFC-3: } \\
\multicolumn{7}{l}{ Mujeres-Varones $(n=372,421)$; AFC-4: Chile-España $(n=793,710)}$.
\end{tabular}

Tabla 2. Análisis de correlaciones entre CMC, atribución del cambio percibido en variables motivacionales y satisfacción con el profesor

\begin{tabular}{|c|c|c|c|c|c|}
\hline & \multicolumn{5}{|c|}{ Cambio percibido en } \\
\hline & Expectativas & Interés & $\begin{array}{l}\text { Habilidad } \\
\text { percibida }\end{array}$ & Esfuerzo & Satisfacción \\
\hline $\begin{array}{l}\text { CMC orientado al } \\
\text { aprendizaje }\end{array}$ & $.658^{* * *}$ & $.710^{* *}$ & $.712^{* *}$ & $.701^{* *}$ & $.731^{* *}$ \\
\hline $\begin{array}{l}\text { Cambio en } \\
\text { expectativas }\end{array}$ & & $.701^{* *}$ & $.684^{* *}$ & $.722^{* *}$ & $.706^{* *}$ \\
\hline Cambio en interés & & & $.745^{* *}$ & $.806^{* *}$ & $.780^{*}$ \\
\hline $\begin{array}{l}\text { Cambio en habilidad } \\
\text { percibida }\end{array}$ & & & & $.771^{* *}$ & $.734^{* *}$ \\
\hline Cambio en esfuerzo & & & & & $.778^{* *}$ \\
\hline
\end{tabular}

muestra chilena completa fueron: $\alpha=.94$ y $\omega=.98$ para CMCQ; $\alpha=.68$ y $\omega=.88$ para expectativas de resultado; $\alpha=.79$ y $\omega=.93$ para interés; $\alpha=.66$ y $\omega=.91$ para habilidad percibida, $\alpha=.73$ y $\omega=.92$ para esfuerzo; y $\alpha=.69$ y $\omega=.92$, para satisfacción con el profesor.

Como muestran las correlaciones que se presentan en la Tabla 2, los alumnos atribuyen en gran medida tanto su satisfacción con el profesor como el grado en que consideran que han mejorado diferentes características personales relacionadas con la motivación al grado en que el profesor genera un clima motivacional orientado al aprendizaje. La varianza explicada por el CMC en relación con las distintas variables oscila entre $37.70 \%$ y $57.76 \%$.

\section{Discusión}

Los resultados obtenidos en la muestra chilena confirman la estructura unifactorial del CMCQ reportada en estudios anteriores, la que se mantiene a través de grupos diferentes, del género y entre las culturas española y chilena. En relación a los estudiantes españoles, en todo caso, los estudiantes chilenos valoran más algunos patrones a la hora de concurrir a un CMC orientado al aprendizaje que sus homólogos españoles. Ello podría estar reflejando las diferencias en las culturas pedagógicas de ambos países. España inició procesos radicales de reforma a comienzos de la década de los 90' (Martin, 2005), incluyendo un fuerte énfasis en la enseñanza centrada en el alumno y el aprender a aprender; mientras que Chile, siguiendo similar énfasis, lo hizo gradualmente a través de programas hasta llegar a una reforma parcial, que no ha contado con adhesión de los docentes y ha sufrido múltiples contratiempos que persisten hasta hoy (Belleï, 2001, 2003; Espinoza \& González, 2012; Silva, s.f.). Así, esta mayor valoración de patrones motivadores ligados a una enseñanza centrada en el aprendizaje, podrían resultar más novedosos y motivadores para los estudiantes chilenos que para los españoles. Por su parte, la confiabilidad y los indicadores de validez predictiva son muy similares a los encontrados en las muestras española, mexicana y francesa (Alonso-Tapia \& Fernández, 2008, 2009; Villasana \& AlonsoTapia, 2015).

Estudio 2: Invarianza factorial entre niveles educativos y validez predictiva en estudiantes chilenos 
La evidencia sobre invarianza factorial del CMCQ entre niveles educativos aportada por Alonso-Tapia y Fernández $(2008,2009)$ muestra que la estructura unifactorial y los pesos de medida de los patrones de actuación docente que lo componen se mantienen. Ambos estudios consideraron las diferencias entre estudiantes de educación secundaria y de bachillerato, lo que no guarda correspondencia con el sistema chileno. En Chile, los grados $7^{\circ}$ y $8^{\circ}$ son considerados parte de la enseñanza primaria o básica (Ministerio de Educación, 1990, 2009), y se agrupan curricular y administrativamente, por tanto, con los grados $5^{\circ}$ y $6^{\circ}$. En consecuencia, se marca un hito importante en el paso entre el $8^{\circ}$ y $9^{\circ}$ grados, mientras hay continuidad entre el $9^{\circ}$ y $12^{\circ}$ grados. En España, en cambio, la enseñanza secundaria contempla un ciclo entre el $7^{\circ}$ y el $10^{\circ}$ grados y luego otro de dos cursos, mientras en México, el primer ciclo contempla los grados $7^{\circ}$ a $9^{\circ}$ (Ministerio de Educación, Cultura y Deporte, 2004; Secretaría de Educación Pública, s.f.). Así, los hitos que marcan cambios significativos en la estructura curricular, que podría afectar la forma en que los estudiantes perciben las actuaciones de sus profesores, es diferente en cada uno de estos países, y sería necesario establecer la invarianza considerando la estructura propia de cada uno, lo que parece particularmente relevante en el caso de Chile, pues aunque en el diseño los dos primeros grados corresponden a la enseñanza secundaria, en la práctica aún operan en la estructura de la educación primaria.

Por otra parte, la validez predictiva del CMCQ se ha establecido claramente en relación con el cambio en variables motivacionales, pero su relación con el rendimiento no ha sido debidamente abordada (Villasana \& AlonsoTapia, 2015). El único estudio en el que se ha incluido el rendimiento ha encontrado una relación estadísticamente significativa pero baja entre el CMC y el rendimiento ( $r=.272$, AlonsoTapia \& Moral, 2010); en ese estudio, no obstante, los participantes fueron estudiantes adultos, lo que podría hacer una diferencia importante con los estudiantes de Educación Secundaria, tanto por razones evolutivas como por la mayor complejidad que representa para los adultos el rol de estudiante, que, en esa etapa, suele coexistir con otros roles y funciones igualmente o más importantes, como el laboral, la pareja o la parentalidad. Concordantemente, en este segundo estudio se propuso: determinar la invarianza por niveles educativos considerando la estructura del sistema educativo chileno; confirmar la invarianza por género; y establecer la relación entre CMC, cambio en variables motivacionales y rendimiento.

\section{Metodología \\ Participantes}

Para este estudio se contó con una muestra de 2701 estudiantes de $7^{\circ}$ a $12^{\circ}$ grados, con edades entre 11 y 19 años $(M=14.08, D T=1.679), 51.2 \%$ mujeres, provenientes de escuelas de las ciudades de Arica e Iquique, Chile. Un tercio (33.3\%) correspondió a los grados $7^{\circ}$ y $8^{\circ}, 36,6 \%$ a los grados $9^{\circ}$ y $10^{\circ}$ y $30,1 \%$ a los grados $11^{\circ}$ y $12^{\circ}$. Esta muestra fue dividida al azar en dos grupos de aproximadamente el mismo tamaño $\left(n_{1}=1342\right.$, $n_{2}=1359$ ), para llevar a cabo análisis multigrupo del modelo de medida del CMCQ por niveles educativos, en el primero, y por sexo, en el segundo. Finalmente, se depuró la muestra descartando los casos que no informaron estimación de rendimiento, para realizar los análisis de modelamiento estructural predictivo; la muestra depurada resultante fue dividida en dos submuestras aleatorias de aproximadamente $25 \%$ $\left(n_{3}=483\right) \quad$ y $75 \% \quad\left(n_{4}=1,517\right)$ de los casos respectivamente, una para realizar el modelamiento, y la otra para establecer la invarianza del modelo estructural entre niveles educativos.

\section{Instrumentos}

Los instrumentos fueron los mismos que en el Estudio 1. Adicionalmente, al solicitar los datos demográficos básicos, se le solicitó a cada estudiante informar acerca de su nota aproximada en la asignatura respecto de la cual se respondía, en la escala de notas usada en Chile ( 1 a 7 , con mínimo de aprobación 4).

\section{Procedimiento}

El procedimiento para administrar el instrumento fue similar al del Estudio 1. La muestra fue conformada por datos recogidos según este procedimiento en el contexto de tres investigaciones diferentes realizadas con 
metodología similar, en que los instrumentos formaron parte de una batería más amplia y diferente en cada caso, en el transcurso de tres años (2011-2013).

\section{Análisis de datos}

Se realizaron AFC multigrupo con estimación por máxima verosimilitud entre niveles educativos con la primera submuestra, dividiendo en tres niveles $\left(7^{\circ}\right.$ y $8^{\circ}, 9^{\circ}$ y $10^{\circ}, 11^{\circ}$ y $12^{\circ}$ grados); y entre géneros con la segunda, después de probar el ajuste del modelo base en cada una de las submuestras. Sobre los datos de la muestra completa se realizaron análisis de correlaciones y análisis de fiabilidad (consistencia interna) con coeficiente Omega de McDonald (1999). Finalmente, se realizó modelamiento estructural de covarianzas para probar el modelo predictivo con la submuestra depurada por información incompleta en las variables dependientes y establecer su invarianza por niveles educativos.

\section{Resultados}

\section{Análisis factoriales confirmatorios}

El estadístico $\chi^{2}$ fue significativo para ambos modelos estimados (AFC-5 y AFC-6), probablemente por el tamaño de las muestras, a las que este indicador es sensible (Hair et al., 2010); pero todos los demás indicadores, incluyendo la razón $\chi^{2} / g l$, caen consistentemente en límites de aceptabilidad de los modelos (para AFC5: $g l=312, \chi^{2}=728.132, p<.001, \chi^{2} / g l=2.334$, $\mathrm{IFI}=.953, \quad \mathrm{CFI}=.953, \quad \mathrm{RMSEA}=.032 ;$ para
AFC6: $g l=208, \chi^{2}=527.399, p=.000, \chi^{2} / g l=2.536$, $\mathrm{IFI}=.964, \mathrm{CFI}=.964$, RMSEA=.034). Cuando se imponen restricciones a los pesos de medida en los análisis multigrupo, el ajuste no se reduce significativamente ni para la comparación entre niveles educativos $\left(\chi^{2}=33.058, g l=30, p=.32\right) \mathrm{ni}$ para la comparación por sexo $\left(\chi^{2}=21.583, g l=15\right.$, $p=.119$ ).

\section{Consistencia interna y correlaciones}

Como se aprecia en la Tabla 3, la consistencia interna del CMCQ fue similar a la observada en los estudios previos; lo mismo ocurre en las escalas de cambio motivacional, en que la consistencia interna está ligeramente por debajo de la reportada en el Estudio 1, pero en cualquier caso es muy buena y del mismo orden que la encontrada en los estudios previos (Alonso-Tapia \& Fernández, 2008, 2009; Villasana \& AlonsoTapia, 2015; Alonso-Tapia, Ruiz, \& Huertas, 2016).

De acuerdo a lo esperado, las correlaciones entre el CMC y cambio en las variables motivacionales son significativas, positivas, y de moderadas a altas, concordantemente con estudios previos (Alonso-Tapia \& Moral, 2010; Villasana \& Alonso-Tapia, 2015). La correlación entre el CMC y la satisfacción con el profesor es también significativa, positiva y moderada, aunque menor que la observada en los estudios previos, en los cuales varió entre $r=.680$ y $r=.823$ (Alonso-Tapia \& Fernández, 2008, 2009; Alonso-Tapia \& Moral, 2010; Villasana \& Alonso-Tapia, 2015).

Tabla 3. Índices Omega $(\omega)$ de consistencia interna y correlaciones entre el CMC orientado al aprendizaje, cambio percibidoen variables motivacionales, satisfacción con el profesor y

\begin{tabular}{|c|c|c|c|c|c|c|c|}
\hline & \multicolumn{5}{|c|}{ Cambio percibido en } & \multirow[b]{2}{*}{ Satisfacción } & \multirow[b]{2}{*}{ Rendimiento } \\
\hline & $\mathrm{CMC}$ & Expectativa & Interés & $\begin{array}{c}\text { Habilidad } \\
\text { percibida }\end{array}$ & Esfuerzo & & \\
\hline $\begin{array}{l}\text { CMC orientado al } \\
\text { aprendizaje }\end{array}$ & .97 & $.545^{* *}$ & $.620^{* *}$ & $.593^{* *}$ & $.621^{* *}$ & $.570^{* *}$ & $.104^{* *}$ \\
\hline $\begin{array}{l}\text { Expectativa de } \\
\text { resultado }\end{array}$ & & .88 & $.646^{* *}$ & $.633^{* *}$ & $.630^{* *}$ & $.612^{* *}$ & $.219^{* *}$ \\
\hline Interés & & & .88 & $.699^{* *}$ & $.741^{* *}$ & $.662^{* *}$ & $.105^{* *}$ \\
\hline $\begin{array}{l}\text { Habilidad } \\
\text { percibida }\end{array}$ & & & & .90 & $.701^{* *}$ & $.645^{* *}$ & $.091^{* *}$ \\
\hline Esfuerzo & & & & & .87 & $.665^{* *}$ & $.121^{* *}$ \\
\hline $\begin{array}{l}\text { Satisfacción con el } \\
\text { profesor }\end{array}$ & & & & & & .91 & $.127^{* *}$ \\
\hline
\end{tabular}


Tabla 4. Estadísticos de bondad de ajuste de modelos SEM, análisis multigrupo por nivel educativo en submuestras aleatorias y análisis por nivel educativo

\begin{tabular}{|c|c|c|c|c|c|c|c|c|c|}
\hline Análisis & $\begin{array}{l}\mathrm{N}^{\circ} \\
\mathrm{Par}\end{array}$ & $\chi^{2}$ & $g l$ & $\mathrm{p}$ & $\chi^{2} / g l$ & GFI & IFI & CFI & RMSEA \\
\hline $\begin{array}{l}\text { SEM-1 (modelo } \\
\text { inicial, } n_{3}=483 \text { ) }\end{array}$ & 48 & 515.159 & 205 & .000 & 2.513 & .91 & .945 & .945 & .056 \\
\hline $\begin{array}{l}\text { SEM-2 (modelo } \\
\text { reespecificado, } \\
n_{3}=483 \text { ) }\end{array}$ & 45 & 517.137 & 208 & .000 & 2.486 & .909 & .946 & .945 & .056 \\
\hline $\begin{array}{l}\text { SEM-3 (modelo } \\
\text { reespecificado, } \\
\text { análisis } \\
\text { multigrupo, } \\
n_{4}=1517 \text { ) }\end{array}$ & 135 & $1,315.036$ & 624 & .000 & 2.107 & .926 & .959 & .959 & .027 \\
\hline $\begin{array}{l}\text { SEM-4 }\left(7^{\circ}-8^{\circ},\right. \\
n=415)\end{array}$ & 45 & 400.808 & 208 & .000 & 1.927 & .918 & .945 & .945 & .047 \\
\hline $\begin{array}{l}\text { SEM-5 }\left(9^{\circ}-10^{\circ},\right. \\
n=588)\end{array}$ & 45 & 485.090 & 208 & .000 & 2.332 & .930 & .959 & .959 & .048 \\
\hline $\begin{array}{l}\text { SEM-6 }\left(11^{\circ}-12^{\circ},\right. \\
n=514)\end{array}$ & 45 & 429.113 & 208 & .000 & 2.063 & .929 & .966 & .965 & .046 \\
\hline
\end{tabular}

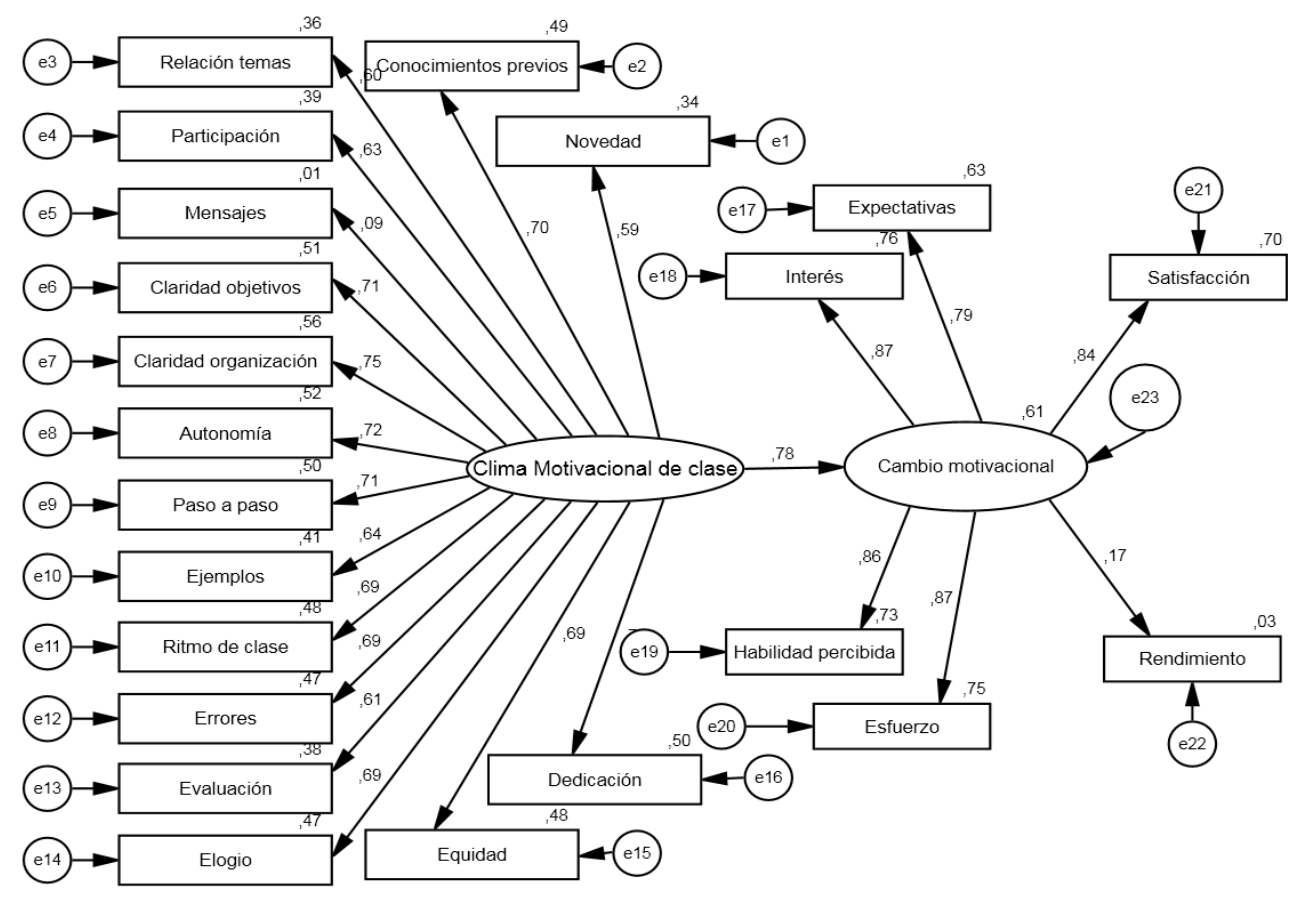

Figura 2. Modelo final predictivo de satisfacción con el profesor y rendimiento (SEM-2).

La correlación entre CMC y rendimiento fue significativa y positiva, aunque baja, menor incluso que la reportada en adultos (Alonso-Tapia \& Moral, 2010). Por su parte, el cambio en las variables motivacionales, también de acuerdo a lo esperado, correlaciona positiva $y$ significativamente con rendimiento, con un tamaño de efecto pequeño, con la excepción del cambio en la expectativa de resultado, cuyo tamaño de efecto podría considerarse medio según el criterio propuesto por Cohen (1988).

\section{Modelamiento estructural}

Para aportar evidencia de la validez predictiva, se llevó a cabo un análisis de covarianzas con ecuaciones estructurales (SEM) en la primera submuestra depurada. En el modelamiento, los cambios motivacionales fueron considerados indicadores de un solo factor, que se denominó cambio motivacional atribuido al profesor. Esto es sostenido por las altas intercorrelaciones entre ellos, $\mathrm{y}$ ha sido sugerido $\mathrm{y}$ utilizado en estudios previos (Alonso-Tapia \& 
Fernández, 2008). Aunque el modelo inicial (que no se muestra por razones de espacio) logró buenos indicadores de ajuste (SEM-1, Tabla 4; Ruiz, Pardo, \& San Martín, 2010), sólo tres de las seis relaciones estructurales postuladas resultaron significativas, por lo que fueron eliminadas, estableciéndose un segundo modelo (Figura 2). Este modelo reespecificado tuvo similar ajuste que el inicial (SEM-2, tabla $4 ; \Delta \chi^{2}=1.978, \Delta \mathrm{gl}=3$, $p=.577$ ), pero es ligeramente más parsimonioso y considera sólo las relaciones significativas.

El modelo así determinado fue sometido a análisis multigrupo con los datos de la segunda submuestra depurada, estableciendo tres grupos según nivel educativo $\left(7^{\circ}-8^{\circ}, 9^{\circ}-10^{\circ}, 11^{\circ}-12^{\circ}\right)$. Este análisis mostró que no hay invarianza entre los grupos $\left(\chi^{2}=27.138, g l=12, p<.01\right)$, por lo que se probó el ajuste del modelo en cada nivel (SEM-3, SEM-4 y SEM-5, Tabla 4), el que fue satisfactorio en cada caso. En la Figura 2 se muestran la proporción de varianza explicada y los coeficientes de regresión estandarizados de las variables endógenas del modelo reespecificado para la submuestra completa.

\section{Discusión}

Los resultados de este segundo estudio confirman la validez del modelo unifactorial del CMCQ y su invarianza a nivel de pesos de medida tanto entre niveles educativos como por sexo en escolares chilenos, así como su consistencia interna; confirman, asimismo, la validez predictiva del CMC respecto de cambio en variables motivacionales y de la satisfacción con el profesor observada en los estudios previos con estudiantes españoles, mexicanos y franceses (Alonso-Tapia \& Fernández, 2008, 2009; AlonsoTapia \& Moral, 2010; Villasana \& Alonso-Tapia, 2015). Respecto de la capacidad del CMC, el cambio motivacional atribuido al profesor y la satisfacción con el profesor para predecir rendimiento, los resultados obtenidos no coinciden exactamente con los reportados por Alonso-Tapia y Moral (2010), pero son congruentes con ellos. En su caso, el CMC explicó una pequeña pero significativa proporción de varianza del rendimiento, mientras que las variables de cambio motivacional no resultaron significativas; en nuestro caso, en cambio, el
CMC no aporta significativamente a la predicción del rendimiento en forma directa, pero sí lo hace la percepción de cambio en las variables motivacionales atribuido al profesor, el que depende, en buena medida, del CMC; esta diferencia en las vías por las que el efecto del CMC se manifiesta en el rendimiento puede deberse a las diferencias metodológicas entre ambos estudios, ya que, en el primer caso, sólo se exploran las relaciones una a una, y en el nuestro, se examina el conjunto de efectos, distinguiendo además los efectos directos de los indirectos. La influencia de estas variables sobre el rendimiento, no obstante, no es similar en los diferentes niveles escolares: su magnitud es mayor en los niveles más bajos de escolaridad, reduciéndose a prácticamente cero en el nivel más alto.

Respecto de la coincidente pequeña proporción de varianza de rendimiento explicada por las variables motivacionales en su conjunto, éste era un resultado esperado, dado que el rendimiento escolar depende de múltiples factores personales, escolares, contextuales, sociales, económicos y culturales (Adeyemy \& Adeyemy, 2014; Payandeh, Najafabadi, \& Farid-Rohani, 2013; Romanik, 2010; Schiefelbein \& Simmons, 1981; Tuñón \& Poy, 2016; Winne \& Nesbit, 2010), por lo que no es extraño que la varianza explicada por solamente algunas de ellas sea pequeña. La diferencia respecto del rol del CMC y las demás variables motivacionales que refleja la ausencia de invarianza del modelo predictivo entre los niveles podría ser explicada por las diferencias entre las muestras, que corresponden a estadios de desarrollo con características muy distintas entre sí. En este sentido, Risso, Peralbo y Barca (2010) encontraron que las variables que predicen el rendimiento cambian a lo largo de la enseñanza secundaria, lo que atribuyen, al menos parcialmente, a cambios evolutivos en ese periodo.

\section{Conclusiones}

Los resultados de ambos estudios presentados muestran la fortaleza del CMCQ para evaluar las pautas de actuación docente que favorecen el CMC orientado al aprendizaje. El modelo unifactorial es plenamente confirmado en los 
estudiantes de educación secundaria chilenos, y la estructura factorial se mantiene invariable a través de género y ciclo dentro de la escolaridad secundaria. Asimismo, se confirma la capacidad del CMCQ para predecir otras variables motivacionales, como los cambios en el interés, la autoeficacia, las expectativas de éxito y el esfuerzo, además de la satisfacción con el profesor. No obstante, también se confirman los hallazgos de Alonso-Tapia y Fernández (2009) y Villasana y Alonso-Tapia (2015), en el sentido de que el contexto cultural influye en el peso relativo que asumen las diferentes pautas de actuación en la conformación del CMC, lo que resulta en que, si bien el modelo unifactorial es replicado interculturalmente, la estructura factorial subyacente no es exactamente la misma en culturas diferentes. Lo anterior implica que el conjunto de estrategias docentes medidos por el CMCQ contribuye a la percepción de un CMC orientado al aprendizaje en estudiantes de distintas culturas, y que los estudiantes que perciben que el CMC facilitado por las pautas de actuación del profesor se orienta al aprendizaje tienden a interesarse y esforzarse más, a sentirse más capaces, a esperar mejores resultados, y a sentirse más satisfechos con el profesor que promueve ese clima en la clase. Sin embargo, la importancia relativa de cada uno de los patrones de comportamiento no es la misma en los distintos contextos culturales. Por ejemplo, los estudiantes franceses valoran más que los españoles el que los profesores promuevan la autonomía (Villasana \& Alonso-Tapia, 2015), mientras que chilenos y españoles no difieren en su valoración de esa pauta; en cambio, los estudiantes chilenos valoran más que sus pares españoles que los profesores evalúen retroalimentando para mejorar a partir de los errores y que actúen equitativamente, entre otras. Estas diferencias no deberían sorprender, ya que diferentes culturas ponen de relieve valores $\mathrm{u}$ orientaciones que dan lugar a formas diferentes de valorar, comportarse y relacionarse (Triandis, 2001), de entender la competencia y la motivación (Plaut \& Markus, 2005) y, como ya hemos mencionado, a culturas pedagógicas diferentes. No obstante, que sean esperables no exime de la tarea de conocerlas y hacerse cargo de ellas, pues para motivar a los estudiantes es necesario tener claridad respecto de cuáles son las pautas que tienen mayor relevancia para ellos a la hora de movilizarlos hacia el aprendizaje.

El principal aporte de este trabajo ha sido contribuir a esclarecer la relación entre el CMC y el rendimiento escolar. $\mathrm{Al}$ respecto, el hallazgo de Alonso-Tapia y Moral (2010) de que el CMC contribuye directamente a explicar el rendimiento de estudiantes adultos, no resulta confirmado en el caso de estudiantes de educación secundaria chilenos. En estos últimos, no es el CMC sino la percepción del cambio en variables motivacionales atribuido al profesor la que se relaciona con el rendimiento. Esta percepción, no obstante, es fuertemente influida por el CMC, y, por esa vía, el efecto directo del cambio en las variables motivacionales sobre el rendimiento es casi enteramente explicado por el efecto indirecto del CMC sobre rendimiento a través del cambio en las variables motivacionales.

También es interesante la diferencia evolutiva de la influencia de la percepción de cambio motivacional atribuido al profesor sobre rendimiento. De acuerdo a ella, podría asumirse que, a medida que los estudiantes avanzan en escolaridad, su rendimiento se hace más independiente del rol motivador del profesor y depende más de otras variables, tal vez del propio estudiante. Esto destaca el rol motivador del profesor en los cursos iniciales de la secundaria; $y$, dentro de ese rol, el que cumple al modelar las expectativas de resultado de sus estudiantes, lo que ha sido remarcado larga e insistentemente (por ejemplo, Chávez, Peinado, Ornelas, \& Blanco, 2013; Valle \& Núñez, 1989).

La baja proporción de varianza del rendimiento explicada por las variables motivacionales, en su conjunto, es un hecho que merece ser comentado. Ya hemos mencionado que una razón para ello puede ser la multiplicidad de factores de los cuales depende. Una razón adicional a considerar es el hecho de que el rendimiento no necesariamente refleja el aprendizaje (Campbell \& Cabrera, 2014), y hay bastante evidencia y fundamento teórico para sostener que la motivación tiene más que ver con el aprendizaje profundo que con el rendimiento (Espinar \& Ortega, 2015; Horzum, Kaymak, \& Gungoren, 2015; Pintrich, Marx, \& Boyle, 1993). Por ello, tal vez sería más justo con el constructo CMC y las variables motivacionales en general, 
relacionarlos con medidas de aprendizaje efectivo y no meramente con rendimiento.

Una limitación de este trabajo que debe ser explicitada es que no se ha realizado con una medida efectiva de rendimiento, sino con un autorreporte del propio estudiante. Probablemente esto tienda a sesgar el resultado, por ejemplo, reduciendo dispersión. Por ello, antes de extraer conclusiones a firme, cabría esperar estudios realizados con medidas de rendimiento efectivo y, adicionalmente, si no mejor, incluyendo también aprendizaje efectivo.

\section{Referencias}

Adeyemy, A. M., \& Adeyemi, S. B. (2014). Personal factors as predictors of students' academic achievement in Colleges of Education in South Western Nigeria. Educational Research and Reviews, 9(4), 97109. doi:http://dx.doi.org/10.5897/ERR2014. 1708

Alonso-Tapia, J., \& Fernández, B. (2008). Development and initial validation of the classroom motivational climate questionnaire (CMCQ). Psicothema, 20(4), 883-889.

Alonso-Tapia, J., \& Fernández, B. (2009). Clima motivacional de clase: Validez transcultural e implicaciones educativas. Infancia $y$ Aprendizaje, 32, 597-612.

Alonso-Tapia, J., Huertas, J. A., \& Ruiz, M. A. (2010). On the nature of motivational orientations: Implications of assessed goals and gender differences for motivational goal theory. The Spanish Journal of Psychology, 13(1), 232-243.

Alonso-Tapia, J., \& Moral, M. A. (2010). Perception of classroom motivational climate in adult non-university students. Psicología Educativa, 16(2), 115-133.doi:http://dx.doi.or $\mathrm{g} / 10.5093 / \mathrm{ed} 2010 \mathrm{v} 16 \mathrm{n} 2 \mathrm{a} 3$

Alonso-Tapia, J., \& Simón, C. (2012). Differences between immigrant and national students in motivational variables and classroommotivational-climate perception. The Spanish Journal of Psychology, 15(1), 61-74. doi:http://dx.doi.org/10.5209/rev_SJOP.2012. v15.n1.37284
Alonso-Tapia J., Nieto C., \& Ruiz, M. (2013). Measuring subjective resilience despite adversity due to family, peers and teachers. The Spanish Journal of Psychology, 16, 1-13.

Alonso-Tapia, J., Panadero, E., \& Ruiz, M. (2014). Development and Validity of the Emotion and Motivation Self-Regulation Questionnaire (EMSR-Q). Spanish Journal of Psychology, 17, 1-15. doi:http://dx.doi.org/10 $.1017 /$ sjp.2014.41

Alonso-Tapia, J., Ruiz, M. A., \& Huertas, J.A. (2016). Differences in classroom motivational climate: causes, effects and implications for teacher education. A multilevelstudy. Manuscritoenevaluación.

Ames, C. (1992). Achievement goals and the classroom motivational climate. En D. H. Schunk y J. L. Meece (Eds.), Students perceptions in the classroom (pp. 327-348). Nueva York: Lawrence Erlbaum.

Belleï, C. (2001). El Talón de Aquiles de la reforma: Análisis sociológico de la política de los 90 hacia los docentes en Chile. En S. Martinic y M. Pardo (Eds.), Economía política de las reformas educativas en América Latina (pp. 227-257). Santiago de Chile: CIDE-PREAL.

Belleï, C. (2003). “¿Ha tenido impacto la Reforma Educativa Chilena?". En C. Cox (Ed.), Políticas educacionales en el cambio de siglo (pp. 125-209). Santiago de Chile: Universitaria.

Bucic, T., \& Robinson, L. (2016). Motivational engagement in the marketing classroom: Individual goal orientations and class climate. Journal of Strategic Marketing. Publicación en línea. doi:http://dx.doi.org/10.1080/09652 54X.2016.1182579

Campbell, C. M., \& Cabrera, A. F. (2014) Making the Mark: Are grades and deep learning related? Research in Higher Education, 55, 494-507.

Chávez, A., Peinado, J., Ornelas, M., \& Blanco, H. (2013). Composición factorial de una escala de autoeficacia en conductas académicas en universitarios de ingeniería. Revista Iberoamericana de Diagnóstico y Evaluación - e Avaliação Psicológica, 35(2), 77-93. 
Clogg, C. C., Petkova, E., \& Haritou, A. (1995). Statistical methods for comparing regression coefficients between models. American Journal of Sociology, 1261-1293.

Cohen, J. (1988). Statistical power analysis for the behavioral sciences ( ${ }^{\circ}$ Ed.). Mahwah, NJ: Erlbaum.

Espinar, R., \& Ortega, J. L. (2015). Motivation: The Road to Successful Learning. PROFILE: Issues in Teachers' Professional Development, 17(2), 125-136.Recuperado de http://eric.ed.gov/?id=EJ1067257

Espinoza, O., \& González, L. E. (2012). Las protestas estudiantiles y sus implicancias para la gestión universitaria en Chile. En N. Fernández (Comp.), La Gestión Universitaria en América Latina (pp. 245-281). Paraguay: Ediciones de la Universidad Nacional de Caaguazú.

Fernández, M. (2004). El clima motivacional de clase en estudiantes adolescentes de Buenos Aires. Revista Iberoamericana de Diagnóstico y Evaluación - e Avaliação Psicológica, 18(2), 119-136.

García-Ripa, M. I., Sánchez-García, M. F., \& Risquez, A. (2016). Estrategias de aprendizaje y autorregulación motivacional. Identificación de perfiles para la orientación de estudiantes universitarios de nuevo ingreso. Revista Iberoamericana de Diagnóstico y Evaluación - e Avaliaçao Psicológica, 41(1), 39-57.

Hair, J. F., Black, W. C., Babin, B. J., \& Anderson, R. E. (2010). Multivariate data analysis. Upper Saddle River, NJ: PearsonPrentice Hall.

Høgheim, S., \& Reber, R. (2016). Supporting interest of middle school students in mathematics through context personalization and example choice. Contemporary Educational Psychology, 42, 17-25. doi:http://dx.doi.org/10.1016/j.cedpsych.2015. 03.006

Horzum, M. B., Kaymak, Z. D., \& Gungoren, O. C. (2015). Structural Equation Modeling Towards Online Learning Readiness, Academic Motivations, and Perceived Learning. Educational Sciences: Theory\&Practice, $\quad$ 15(3), 759770.10.12738/estp.2015.3.2410
Martin, E. (2005, octubre 3). La LOGSE, 15 años después. El País (España). Recuperado desde http://elpais.com/diario/2005/10/03/educacion /1128290409_850215.html.

McDonald, R. P. (1999). Test theory. A unified treatment. Mahwah, NJ: Lawrence Erlbaum.

Meece, J. L., Anderman, E. M., \& Anderman, L. H. (2006). Classroom goal structure, student motivation, and academic achievement. Annual Review of Psychology, 57, 487-503.

Ministerio de Educación. (1990). Ley $N^{\circ} 18.962$. Ley Orgánica Constitucional de Enseñanza. Santiago de Chile: Ministerio.

Ministerio de Educación. (2009). Ley $N^{\circ} 20.370$. Establece la Ley General de Educación. Santiago de Chile: Ministerio.

Ministerio de Educación, Cultura y Deporte. (2004). El sistema educativo español. Madrid: MECD/CIDE.

Ministerio de Educación, Cultura y Deporte. (s.f.). Enseñanzas del sistema educativo. Madrid: Ministerio.http://www.mecd.gob.es/educacion -mecd/areas-educacion/sistema-educativo/en senanzas.html

Núñez, J. C., Vallejo, G., Rosario, P., Tuero, E., \& Valle, A. (2014). Student, teacher, and school context variables predicting academic achievement in Biology: Analysis from a multilevel perspective. Revista de Psicodidáctica, 19(1), 145-171.

Patall, E. A., Vasquez, A. C., Steingut, R. R., Trimble, S. S., \& Pituch, K. A. (2016). Daily interest, engagement, and autonomy support in the high school science classroom. Contemporary Educational Psychology, 46, 180-194. doi:http://dx.doi.org/10.1016/j.cedps ych.2016.06.002

Payandeh, A. T., Najafabadi, M. O., \& FaridRohani, M. R. (2013). Factors contributing to academic achievement: A Bayesian Structure Equation Modelling Study. International Journal of Mathematical Education in Science and Technology, 44(4), 490-500. doi: http://dx.doi.org/10.1080/0020739X.2012.742 149

Pintrich, P. R., Marx, R. W., \& Boyle, R. A. (1993). Beyond cold conceptual change: The role of motivational beliefs and classroom contextual factors in the process of conceptual change. Review of Educational Research, 
63(2), 167-199. Recuperado de http://www.jstor.org/stable/1170472

Plaut, V. C., \& Markus, H. R. (2005). The "Inside" Story. A cultural-historical analysis of being smart and motivated, american style. En Elliot, A. y Dweck, C., Handbook of competence and motivation (pp. 457-488). NY: Guilford.

Raghul, E., \& Saradha, R. (2016). Creating a positive classroom climate: A motivational factor for English language acquisition. Man in India, 96(5), 1459-1465. Resumen recuperado de http://www.scopus.com/

Risso, A., Peralbo M., \& Barca, A. (2010). Cambios en las variables predictoras del rendimiento escolar en Enseñanza Secundaria. Psicothema, 22(4), 790-796.

Romanik, D. (2010). Out-of-school factors affecting academic achievement. Information Capsule, 1004, 1-17. Recuperado de http://eric.ed.gov/?id=ED536510

Ruiz, M. A., Pardo, A., \& San Martín, R. (2010). Modelos de ecuaciones estructurales. Papeles del Psicólogo, 31(1), 34-45.

Salili, F., \& Hoosain, R. (Eds.). (2007). Culture, motivation and learning: A multicultural perspective. Charlotte, NC: IAP.

Schiefelbein, E., \& Simmons, J. (1981). The determinants of school achievement: A review of the research for developing countries (Report N ${ }^{\circ}$ IDRC-TS24e). Ottawa (Ontario), Canadá: International Development Research Centre. Resumen disponible en www.ebscohost.com

Schwinger, M., Steinmayr, R., \& Spinath, B. (2016). Achievement goal profiles in elementary school: Antecedents, consequences, and longitudinal trajectories. Contemporary Educational Psychology, 46, 164-179. doi:http://dx.doi.org/10.1016/j.cedp sych.2016.05.006

Secretaría de Educación Pública. (s.f.). La estructura del sistema educativo mexicano. Recuperado desde http://www.sep.gob.mx/wo rk/models/sep1/Resource/1447/1/images/siste maedumex09_01.pdf

Silva, S. (s.f.). La "revolución pingüina" y el cambio cultural en Chile. Recuperado desde http://www.archivochile.com/carril_c/cc2012/ cc2012-062.pdf
Simón, C., \& Alonso-Tapia, J. (2016). Positive classroom management: Effects of disruption management climate on behaviour and satisfaction with teacher. Revista de Psicodidáctica, 21(1), 65-86. doi:10.1387/Rev Psicodidact.13202

Triandis, H. C. (2001). Individualism-collectivism and personality. Journal of Personality, 69(6), 907-925.

Tuñón, I., \& Poy, S. (2016). Factores asociados a las calificaciones escolares como proxy del rendimiento educativo. Revista Electrónica de Investigación Educativa, 18(1), 98-111. Recuperado desde http://redie.uabc.mx/redie /article/view/615

Urdan, T., \& Turner, J. C. (2005). Competence motivation in the classroom. En A. J. Elliot y C. Dweck (Eds.), Handbook of competence and motivation (pp. 297-317). Nueva York: Guilford.

Valle, A., \& Núñez, J. C. (1989). Las expectativas del profesor y su incidencia en el contexto institucional. Revista de Educación, 290, 293319.

Villasana, M., \& Alonso-Tapia, J. (2015). Validez transcultural del «Cuestionario de Clima Motivacional de Clase»: Comparación entre estudiantes franceses y españoles. Revista de Psicodidáctica, 20(2), 227-246.

Winne, P. H., \& Nesbit, J. C. (2010). The psychology of academic achievement. Annual Review of Psychology, 61, 653-78. 